\title{
Point Prevalence Survey of Antibiotic Prescribing in the Hospital Sector in Albania
}

\author{
Silvi BOZO" ${ }^{*}$, Carlo De Giuli MORGHEN1', Elena CAPPARELLI', Eftiola POJANI² \\ 1 Department of Chemical-Pharmaceutical and Biomolecular Technologies, Faculty of Pharmacy, Catholic University "Our \\ Lady of Good Counsel", Tirana, Albania. \\ 2Department of Pharmaceutical Sciences, Faculty of Pharmacy, Catholic University Our Lady of Good Counsel, Tirana, \\ Albania.
}

\begin{abstract}
Antibiotic resistance represents a serious threat worldwide. The onset and spread of resistance is mostly related to irrational and increased use of antibiotics. The aim of this study was to evaluate antibiotic prescribing patterns and identify areas for quality improvement in the hospital sector in Albania. A Point Prevalence Survey study was conducted, at a University Hospital Centre in Tirana, Albania. On the day of the survey, $65.7 \%$ of the inpatients were on antibiotic treatment, mostly for medical prophylaxis (67.1\%). The higher prevalence was found in Intensive Care Units (81.0\%). The most commonly used antibiotics were the cephalosporins (53.1\%) followed by metronidazole (16.2\%) and fluoroquinolones (14.7\%). High rates of antibiotic use were found in all wards, while some indicators related to prescribing patterns were critical. A close and continuous surveillance of the antibiotic use, along with measures at national level could contribute in improving a proper use of antibiotics.
\end{abstract}

Key words: prevalence survey, antibiotic use, Albania, antibiotic surveillance.

\section{INTRODUCTION}

Antibiotics are among the most frequently prescribed drugs worldwide. According to the studies carried out in the European countries and the United States, $23-38 \%$ of inpatients are on systemic antibiotic treatment ${ }^{1}$. Moreover, from the data collected worldwide it appears that the consumption of antibiotics is constantly increasing and in the years 2000-2010 there was an increase of $36 \%$ in their global consumption ${ }^{2}$. One of the main causes for the onset and

\footnotetext{
*Corresponding Author: s.bozo@unizkm.al ORCIDs:

Silvi Bozo : : : https://orcid.org/0000-0002-6444-0799

Carlo De Giuli Morghen : https://orcid.org/0000-0002-4127-3596

Elena Capparelli : : https://orcid.org/0000-0002-7031-4422

Eftiola Pojani : : https://orcid.org/0000-0001-5402-4065

(Received 24 Jan 2020, Accepted 29 March 2021)
} 
diffusion of antibiotic resistance is their increased and irrational consumption and administration. This correlation is well documented in the medical literature ${ }^{3,4,5,6,7}$. Antibiotic resistance has a significant impact on patients morbidity, mortality, occurrence of side effects, toxicity and prolonged hospitalization. Another important consequence is the increased costs incurred by government, patients, insurance companies, or other third-parties when antibiotics are misused. A large portion of antibiotic use appears to be for viral or spontaneously resolving bacterial infections. The Centers for Disease Control and Prevention (CDC) estimates that about 100 million courses of antibiotics are prescribed by office-based physicians each year, and that approximately one-half of those prescriptions are unnecessary ${ }^{8,9,10,11}$. Studies evaluating physicians' prescribing patterns have found that many of these antibiotic courses are unnecessary, with a projected 40\%-60\% of patients receiving antibiotic treatment for viral upper respiratory tract infections. This prescribing pattern persists despite the fact that antibacterial agents have no significant benefit for the resolution of viral diseases, such as the common cold ${ }^{12,13,14,15,16}$.

The hospital sector, with an increased antibiotic consumption and a facilitated diffusion of resistance, is a major driver of the spread of multidrug-resistant bacteria. In fact, patients who are hospitalized for prolonged periods are more at risk of acquiring antibiotic-resistant nosocomial infections, which are also very difficult to treat and lead to increased treatment costs ${ }^{17}$. This may also require the use of innovative and more expensive antibiotics which may not be readily available. Some of these second-line antibiotics are not necessarily more effective than first line agents but are the last line of antibacterial defense. The courses of treating resistant microorganisms are usually longer than the courses of treating susceptible microorganisms ${ }^{18,19}$.

Antibiotic prescribing patterns differ not only between different countries, but also between hospitals of a same country. These differences may be related to hospital and patient features, antibiotic policies at a hospital level, prescribing physician preferences and differences in public education and health systems ${ }^{2}$.

Antibiotic resistance is a global problem, affecting both developing and developed countries. Especially in developing countries there is lack of centralized systems for monitoring resistance patterns and antibiotic consumption ${ }^{20,21,22}$. In fact, in Albania, the data on trends of resistance and antibiotic consumption are scarce and fragmentary and there is a lack of consistent representative data at national level. The aim of the present work was to evaluate antibiotic consumption, investigate antibiotic prescribing patterns and identify areas for quality improvement in the hospital sector in Albania towards a more rational use of antibiotics. 


\section{METHODOLOGY}

\section{Study design}

The study was carried out at the University Hospital Center "Mother Teresa" (QSUT) located in the capital of Albania, Tirana. QSUT is the only university and tertiary hospital in Albania. Moreover, this hospital represents a reference center for all the regional State hospitals and therefore can be considered a representative institution for the use and consumption of antibiotics nationwide.

For the evaluation of antibiotic consumption, a Point Prevalence Survey (PPS) was conducted in May 2017, including 11 wards (2 medical wards, 5 surgery wards and 4 Intensive Care Units, ICU) with a total 356 bed capacity. Only adult patients were enrolled in the study.

\section{Data collection}

Data were collected mainly by consulting patient prescribing charts, supplemented by information acquired by the Hospital Information System, laboratory data and interviews with physicians, nurses and pharmacists within the hospital, using an ad-hoc method according to the Global PPS methodology ${ }^{23}$. A patient form was completed only for those patients on antibiotic treatment at 8 o'clock on the day of the survey. Only data related to antibiotics for systemic use included in the Anatomical Therapeutic Classification, ATC class Jo1, were collected $^{24}$. Antibiotics for topical use were excluded from the study.

However, all patients whether or not on antibiotic treatment were counted in the denominator data. In our study were enrolled a total of 315 patients, corresponding to the number of patients admitted to each of the wards included on the specific study day.

Overall, bed occupancy rate was 88.5\% (315/356). The collected data included age, gender, type of antibiotic, dosage, reasons and indications for treatment, as well as compliance to guidelines. All patients were de-identified and to every patient record was given a unique not identifiable survey number.

\section{Statistical analysis}

Data were analyzed using the SPSS version 19.0 software. Chi-square tests were used for comparing the percentages between variables. P-value $\leq 0.05$ was considered as significant. 


\section{RESULTS AND DISCUSSION}

On the day of the survey, 207 (65.7\%) out of 315 total inpatients were on antibiotic treatment for any reason. The overall bed occupancy rate was $88.5 \%$ (315/356 beds). Distribution rates of antibiotic use and bed occupancy rate according to the ward type are presented in Table 1.

Table 1. Distribution rates of antibiotic use and bed occupancy rate according to the ward type

\begin{tabular}{|c|c|c|c|c|}
\hline Ward type & No. of beds & $\begin{array}{c}\text { No. of } \\
\text { inpatients }\end{array}$ & $\begin{array}{c}\text { Bed occupancy } \\
\text { rate (\%) }\end{array}$ & Prevalence $^{*}$ \\
\hline \multicolumn{5}{|c|}{ Medical } \\
\hline Infectious diseases & 75 & 68 & 90.7 & $57 / 83.8$ \\
\hline Hematology & 34 & 34 & 100.0 & $17 / 50$ \\
\hline Total & 109 & 102 & 93.6 & $74 / 72.5$ \\
\hline \multicolumn{5}{|c|}{ Surgery } \\
\hline General Surgery, Clinic 1 & 35 & 35 & 100.0 & $16 / 45.7$ \\
\hline Urology & 45 & 40 & 88.9 & $38 / 95.0$ \\
\hline General Surgery, Clinic 3 & 51 & 51 & 100.0 & $35 / 68.6$ \\
\hline Neurosurgery & 32 & 24 & 75.0 & $1 / 4.2$ \\
\hline Burn and plastic surgery & 33 & 21 & 63.6 & $9 / 42.8$ \\
\hline Total & 196 & 171 & 87.2 & $99 / 57.9$ \\
\hline \multicolumn{5}{|c|}{ Intensive Care Unit (ICU) } \\
\hline Infectious diseases & 5 & 2 & 40.0 & $2 / 100.0$ \\
\hline Central ICU & 15 & 14 & 93.3 & $14 / 100.0$ \\
\hline Cardiology & 21 & 21 & 100.0 & $13 / 61.9$ \\
\hline Neurosurgery & 10 & 5 & 50.0 & $5 / 100.0$ \\
\hline Total & 51 & 42 & 82.3 & $34 / 81.0$ \\
\hline
\end{tabular}

*Prevalence is expressed as the ratio of No. of antibiotic-treated patients/ the percentage of patients under antibiotic treatment

* chi - square test; the highest rate of antibiotic use was observed in the ICU wards $(\chi 2=2.3 p=0.5)$

In our study, the prevalence of antibiotic use is relatively high, if compared to the other European countries. According to the Global PPS report of 2015, the prevalence of antibiotic use in Europe is of $31.1 \%^{26}$. The prevalence rate in our study is higher compared even with countries reported with the highest prevalence of antibiotic use, respectively West \& Central Asia (42.1\%), followed by 
South America (39.5\%), Australia and New Zealand (38.5\%), North America (35.2\%), East \& South Asia (33.3\%) ${ }^{23}$. Different rates can also be seen depending on the country and the hospital in which other studies are conducted. For instance, prevalence rates similar to our study were reported in a study performed in four tertiary hospitals in Nigeria (69.7\%), in 11 hospitals from the Democratic Republic of Congo (68\%) and some other developing countries ${ }^{26,27}$. However it's important to note that QSUT is a reference center for all regional hospitals in Albania, where are hospitalized patients suffering from serious infectious diseases that cannot effectively be treated in the hospital of origin. Therefore, the high levels of prevalence of antibiotic consumption deriving from the data of our study could be partly explained from a presumed high prevalence of patients affected by bacterial infections in this hospital.

The prevalence levels of antibiotic consumptions found in our study were higher if compared also with previous data from Albania. The data reported from an Albanian study performed as part of the Global PPS project of 2015, where were involved 3 Albanian hospitals (including the QSUT), show a lower level of prevalence (47.5\%) compared to our study ${ }^{23}$. Higher values also result in comparison with the prevalence levels reported in another PPS study conducted in Albania in the QSUT in 2003, where the prevalence was $46.9 \%{ }^{28}$.

The differences observed between different studies carried out in Albania may be due to the number of wards of the various specialties included in the study, considering that in different specialties, the rate of administration of antibiotics presents considerable differences, as well as the period and the temporal distance between the studies. In our case, sampling was of convenience, and therefore were included in the study those wards were we expected to find the highest rate of antibiotic administration. In other studies previously conducted in Albania, the goal was to include as many wards as possible, but without paying particular attention to the quality and types of wards in terms of antibiotic consumption levels.

The highest rate of antibiotic use in our study was observed in the ICU wards $(\chi 2=2.3 p=0.5)$, ranging from $61.9 \%$ in the cardiology ICU to $100 \%$ in the infectious diseases, central and neurosurgery ICU. This could be partially related to the debilitated physical condition and deficiencies of the immune system in the patients in these units which require intense antibiotic therapy for longer periods and for severe infectious complications, including nosocomial infections. In fact, a study conducted in the Danish hospitals estimated that the total antibiotic consumption in the ICUs is approximately tenfold greater than in general hospital wards ${ }^{29}$. However, high levels of prevalence in our study were also found in the medical (72.5\%) and surgery wards (57.9\%), although not comparable with the ICU wards. 
The treated patients (207) received a total of 327 antibiotics (1.6 antibiotics/patient). In $53.1 \%$ of the patients receiving antibiotic therapy, was administered only one antibiotic, while in $46.9 \%$ of patients the therapy consisted of 2 or 3 antibiotics simultaneously. None of the patients were given more than 3 antibiotics at the same time.

The types of indication were defined and classified according to the GLOBAL PPS protocol, respectively as Community-acquired infection (CAI), Hospitalacquired infection (HAI), Medical Prophylaxis (MP), Surgical Prophylaxis (SP) or Unknown indication ${ }^{23}$. The most common indication for antibiotic use (67.1\% of the patients), was medical prophylaxis $\left(\chi^{2}=197 \mathrm{p}<0.01\right)$, followed by community-acquired infection (CAI) (26.6\%), hospital-acquired infection (HAI) (4.8\%) and surgical prophylaxis (2.9\%) (Table 2).

Table 2. Distribution rates of antibiotic use according to the type of indication

\begin{tabular}{|c|c|c|c|c|}
\hline \multirow{2}{*}{\begin{tabular}{c} 
Type of indication \\
\cline { 2 - 4 }
\end{tabular}} & \multicolumn{3}{|c|}{ No./\% of patients } \\
\cline { 2 - 5 } & Medical wards & Surgical wards & (CU & Total $^{*}$ \\
\hline $\begin{array}{c}\text { Community-acquired infection } \\
\text { (CAI) }\end{array}$ & $46 / 62.2$ & $5 / 5.1$ & $4 / 11.8$ & $55 / 26.6$ \\
\hline $\begin{array}{c}\text { Hospital-acquired infection } \\
\text { (HAI) }\end{array}$ & $4 / 5.4$ & $3 / 3.0$ & $3 / 8.8$ & $10 / 4.8$ \\
\hline $\begin{array}{c}\text { Medical Prophylaxis (MP) } \\
\text { Surgical Prophylaxis (SP) }\end{array}$ & $25 / 33.8$ & $89 / 89.9$ & $25 / 73.5$ & $139 / 67.1$ \\
\hline Unknown indication & $2 / 2.7$ & $6 / 6.1$ & $0 / 0$ & $6 / 2.9$ \\
\hline
\end{tabular}

$+\S \star^{*}$ chi-square test;

${ }^{+}$In medical wards, the most common indication for antibiotic use was CAI $(\chi 2=197 p<0.01)$.

${ }^{\text {sI }} \mathrm{n}$ surgical wards, the most common indication for antibiotic use was MP $(\chi 2=197 p<0.01)$.

${ }^{*}$ In ICU wards, the most common indication for antibiotic use was MP ( $\chi^{2}=197$ $p<0.01)$.

"In the total sample, the most common indication for antibiotic use was MP $(\chi 2=197 p<0.01)$.

Medical Prophylaxis is classified as the indication when the antibiotic therapy is used as general prophylaxis, without targeting a specific site, to prevent HAIs and/or surgical site infections, but in any case without the presence of 
any identified or confirmed infection. HAIs included post-operative surgical site infection (30.0\%), intervention related infections (30.0\%) such as Ventilator Associated Pneumonia (VAP) or Catheter related-Urinary Tract Infection (C-UTI) or other hospital acquired infections such as Hospital Acquired Pneumonia (HAP). On the other hand, prolonged surgical prophylaxis for more than one day, was very common. These results are similar to other studies conducted in Albania with a relatively high percentage of patients (67.1\%) who received antibiotic therapy as medical prophylaxis ${ }^{23}$. If compared to European data, this percentage is almost 10 times the relative value reported by the ESAC 2006 study conducted in 20 European hospitals $(6.6 \%)^{14}$. In our study, the surgical departments are those with the highest proportion of patients under antibiotic therapy as medical prophylaxis (89.9\%), followed by ICU departments (73.5\%). This result could partially be justified by the critical conditions of the patients admitted to these wards, with a high sensitivity to nosocomial infections. However, in many cases, the administration of the antibiotic therapy as a medical prophylaxis in the patients of our study did not have a precise and/ or controlled well-based clinical justification. From the information received from the hospital's healthcare staff, the administration of antibiotics throughout the patient's hospitalization turns out to be a common practice for physicians. This suggests that initiatives needs to be taken in order to strengthen infection preventive practices and reduce associated complications, This would improve physicians confidence in infection prevention and control programs, which would lead to substantial reductions and more rational use of antibiotics in the hospital setting as well. Type, frequency of antibiotic use and the ATC code for each antibiotic and class is shown in Table 3. 
Table 3. Type and frequency of antibiotics

\begin{tabular}{|c|c|c|c|}
\hline ATC code & Antibiotic & No. of patients & Frequency of use (\%)* \\
\hline \multirow[t]{3}{*}{ J01A } & Tetracyclines & 4 & 1.2 \\
\hline & Tigecycline & 2 & 0.6 \\
\hline & Doxycycline & 2 & 0.6 \\
\hline J01B & Amphenicols & 0 & 0 \\
\hline \multirow[t]{3}{*}{ J01C } & Beta-lactam antibacterials, penicillins & 3 & 0.9 \\
\hline & Piperacillin & 1 & 0.3 \\
\hline & Piperacillin and Tazobactam & 2 & 0.6 \\
\hline \multirow[t]{8}{*}{ J01D } & Other beta-lactam antibacterials & 174 & 53.2 \\
\hline & Cefazolin & 91 & 27.8 \\
\hline & Ceftriaxone & 41 & 12.5 \\
\hline & Cefuroxime & 24 & 7.3 \\
\hline & Cefepime & 12 & 3.7 \\
\hline & Ceftazidime & 2 & 0.6 \\
\hline & Cefotaxime & 2 & 0.6 \\
\hline & Cefalexine & 2 & 0.6 \\
\hline \multirow[t]{2}{*}{ J01E } & Sulfonamides and trimethoprim & 5 & 1.5 \\
\hline & Sulfamethoxazole and trimethoprim & 5 & 1.5 \\
\hline J01F & $\begin{array}{l}\text { Macrolides, lincosamides and } \\
\text { streptogramins }\end{array}$ & 0 & 0 \\
\hline \multirow[t]{3}{*}{ J01G } & Aminoglycoside antibacterials & 36 & 11.0 \\
\hline & Gentamicin & 23 & 7.1 \\
\hline & Amikacin & 13 & 4.0 \\
\hline \multirow[t]{3}{*}{ J01M } & Quinolone antibacterials & 48 & 14.7 \\
\hline & Ciprofloxacin & 25 & 7.7 \\
\hline & Levofloxacin & 23 & 7.1 \\
\hline J01R & Combinations of antibacterials & 0 & 0 \\
\hline \multirow[t]{3}{*}{ J01X } & Other antibacterials & 57 & 17.5 \\
\hline & Metronidazole & 53 & 16.2 \\
\hline & Vancomycin & 4 & 1.2 \\
\hline
\end{tabular}

* chi-square test;

The class of antibiotics used most frequently is the Jo1D group (Other betalactams) $\left(\chi^{2}=468 p<0.001\right)$.

Among all antibiotics, the most frequently used antibiotic is cefazolin ( $\chi 2=987$ $p<0.001)$. 
The results of our study show that among the antibiotics of the ATC class Jo1, the class of antibiotics used most frequently is the Jo1D group (Other betalactams) (53.2\%) ( $\left.\chi^{2}=468 \mathrm{p}<0.001\right)$, followed by the Jo1X group (Other antibacterials) (17.5\%), the Jo1M group (Quinolones) (14.7\%) and finally the Jo1G group (Aminoglycosides) (11.0\%).

Cefazolin was found to be the most frequently used among all antibiotics (27.8\%) ( $\mathrm{p}<0.001)$ followed by metronidazole (16.2\%) and ceftriaxone (12.5\%). These results are consistent with those reported for Albania in Global PPS, except for metronidazole where, the frequency of use in our study is higher ${ }^{23}$. Similar results are found also in a PPS study involving 13 Chinese hospitals ${ }^{30}$. On the other hand, different prescription trends are reported in other studies $^{13,31,32}$. In a large-scale PPS study at European level, the combination of penicillins with a beta-lactamase inhibitor (24\%) was the most widely used class of antibiotics, followed by macrolides (15\%), fluoroquinolones (11\%) and thirdgeneration cephalosporins $(10 \%)^{14}$. In another study conducted in 130 U.S. hospitals, it appeared that the classes of antibiotics most frequently administered to adults were fluoroquinolones, cephalosporins, metronidazole and vancomy$\operatorname{cin}^{33}$. Globally, it appears that high levels of cephalosporin use, similar to our study, are found in Russia (65.8\%) and Serbia (52.9\%) ${ }^{34}$.

The prescription practices observed in our study, with a large use of broadspectrum antibiotics could be attributed to the fear for inadequate antibiotic coverage by the physicians. Changes in antibiotic use in hospitals undergoing these studies can also be attributed to differences in microbial resistance trends between countries, the presence and implementation of guidelines, the duration of empirical therapy, the clinical conditions of the patients and the common habits in prescribing this class of drugs ${ }^{30}$. Furthermore, we have observed that the antibiotics choice was also conditioned by their availability and cost in the hospital setting rather than by the clinical needs of the patients. As a matter of fact, since Albania is a still developing country and the healthcare system has limited financial resources, the availability of a wide class of antibiotics is very limited. In fact, antibiotics such as carbapenems do not appear to be administered in any of the patients in our study and antibiotics such as glycopeptides (vancomycin) have been used in a very small number of patients due to their prohibitive cost for the hospital and the patient.

Different trends in antibiotic use were observed across the different wards in our study. For instance, in the medical wards, the most widely used antibiotic is cefuroxime (19.1\%). The antibiotics used in surgical wards, in decreasing order of frequency are cefazolin (37.6\%), metronidazole (24.4\%) and ceftriaxone (8.8\%). 
Unlike the data on the total sample, in these wards there is also a high percentage of gentamicin prescriptions, while in ICUs, the most widely used antibiotic is ceftriaxone (34.7\%), followed by cefazolin (24.5\%) and ciprofloxacin (12.3\%). Compared to the other specialties, the variety of antibiotics used in the ICU is generally lower.

Several hospital quality indicators regarding antibiotic prescription were also evaluated and the results according to the ward type and the total sample are shown in Table $4^{13}$. The results are represented as the number and percentage of antibiotics on the total number of antibiotics administered on the day of PPS.

Table 4. Hospital quality indicators for antibiotic use

\begin{tabular}{|c|c|c|c|c|}
\hline \multirow{2}{*}{ Parameter description } & \multicolumn{3}{|c|}{ No./\% of antibiotics } \\
\cline { 2 - 5 } & Medical wards* & Surgical wards & ICU* & \multirow{2}{*}{ Total $^{*}$} \\
\hline Targeted treatment & $8 / 7.3$ & $2 / 1.2$ & $0 / 0.0$ & $10 / 3.1$ \\
\hline Guideline compliance & $83 / 75.4$ & $2 / 1.2$ & $6 / 12.2$ & $91 / 27.8$ \\
\hline Treatment based on biomarker data & $55 / 50.0$ & $30 / 17.9$ & $6 / 12.2$ & $91 / 27.8$ \\
\hline Reason written in notes & $41 / 37.3$ & $75 / 44.6$ & $10 / 20.4$ & $126 / 38.5$ \\
\hline
\end{tabular}

* chi-square test; $p<0$. oo1

Data indicate a remarkably high proportion of empirically administered antibiotics (96.9\%), compared to those administered according to microbiological findings (3.1\%) $(\mathrm{p}<0.001)$. Results are similar to other studies performed in Albania as well as in other low-income countries such as Congo (neither of the hospitalized patients received targeted therapy) and Mongolia (targeted therapy was prescribed in $9.2 \%$ of the cases) ${ }^{27,35}$. Conversely, the European countries show a significantly higher prevalence of targeted prescriptions. For instance, in the ESAC 2009 study, the average percentage is $43 \%$, while in many Swedish hospitals it reaches levels of $70 \%{ }^{14,36}$.

In our study, the specialties with the highest rate of empirical therapy were the ICUs. Especially in patients with a severe clinical condition, it is well known that initiation of empiric therapy is associated with an increased survival rate of critically ill patients, and therefore some authors suggest empiric therapy with antibiotics (alone or in combination) in the case of serious bacterial infections ${ }^{37}$. Nevertheless, the revision of therapy in the ICU should be carried out on the third day of therapy to review the therapy used after the outcome of the microbiological examination (de-escalation) ${ }^{38}$. Therefore, the analysis of the patient's microbiological cultures remains a fundamental requirement to ensure patient safety, especially when it comes to hospital acquired infections that are often caused by multi-drug resistant bacteria pathogens. 
In Albania there are no prescription guidelines for the administration of antibiotics, neither at a national nor a local level. As a result, at the QSUT no protocols and guidelines were found that would serve as a reference for comparing the concordance of antibiotic therapy found for each individual patient on the day of the study. Thus, since in our study the treatment was considered appropriate if prescribed by an infection specialist, the results showed that guideline compliance occurred only in $27.8 \%$ of the prescriptions $(\mathrm{p}<0.001)$. Clinical guidelines should therefore be considered crucial to improve the quality of antibiotic prescriptions, both to promote the effectiveness of empirical therapy and to reduce the inappropriate use of antibiotics in the ongoing fight against resistant pathogens. The absence of clinical guidelines in Albania, highlights the need for national and local guideline design and implementation that can assist the physician in taking appropriate decisions in specific clinical settings and pathologies.

On the other hand, treatment was based on biomarker data in $27.8 \%$ of the prescriptions and the mainly biomarker was the C-reactive protein (PCR) (15.3\%) ( $p<0.001)$. Other biomarkers used were the erythrocyte sedimentation index, the HIV-related immune dysfunction, the brucellosis and the cerebrospinal fluid biomarkers.

The reason for which antibiotics were prescribed was recorded in $38.5 \%$ of the patients medical records. Moreover, in many of these cases, the physician's notes were unclear and it was often necessary to refer to the entire patient's medical history.

In conclusion, this study reported high rates of antibiotic use in Albanian hospitals. In addition, some other indicators related to the patterns of prescribing were critical, such as lack of microbiology data, low documentation of the reasons for treatment, its duration, and the lack of hospital guidelines. This situation is problematic also in comparison with other industrialized countries or developing countries and the data reported at a regional and international level.

The present study has some limitations such as the data bias due to the selection bias and the type of wards included in the study which could provide unrepresentative data at a hospital level. The partial lack of information in the patient charts encountered in some cases, may have also influenced the data represented for some indicators related to the antibiotic prescriptions. However, this research, was performed in accordance with the guidelines of the Global PPS methodology, contributing to provide useful information regarding the use of antibiotics in the hospital setting and highlighted some differences with other studies previously conducted in Albania. 
The results of our study, as well as those from previous ones conducted in Albania, suggest that more guidance on the use of antibiotics for the treatment of infections or prophylaxis is needed as well as policies of antibiotic use in the hospitals in terms of more rational prescriptions. A close and continuous surveillance of the antibiotic use, as part of the structuring of policies, along with measures at national level such as development of local guidelines and continuous education, could contribute to improving the appropriate and proper use of antibiotic therapy. These results need further investigation through qualitative or quantitative studies.

\section{STATEMENT OF ETHICS}

Approval was obtained from the Republic of Albania Medical University of Tirana Ethical Council prot. nr. 2104/1 on 07/09/2021.

\section{ACKNOWLEDGEMENT}

We would like to express our gratitude to A. Radaelli, Department of Medical Biotechnologies and Translational Medicine, State University of Milano, Italy for lexically revising the manuscript. 


\section{REFERENCES}

1. The European Centre for Disease Prevention and Control (ECDC). Available from: http:// ecdc.europa.eu/en/eaad/Documents/antibiotics-consumption-EU-data-2014.pdf.

2. Van Boeckel T.P, Gandra S, Ashok A, Caudron Q. Grenfell B.T, Levin S.A, et al. Global antibiotic consumption 2000 to 2010: an analysis of national pharmaceutical sales data. Lancet Infect Dis, 2014; 14(8): 742-750.

3. Pouwels K, Freeman R, Muller-Pebody B, Rooney G, Henderson K, Robotham L, et al. Association between use of different antibiotics and trimethoprim resistance: going beyond the obvious crude association. J Antimicrob Chemother, 2018; 73: 1700-1707.

4. Klein E.Y, Van Boeckel T.P, Martinez E.M, Pant S, Gandra S, Levin S.A, et al. Global increase and geographic convergence in antibiotic consumption between 2000 and 2015. Proc Natl Acad Sci U S A, 2018; 115(15): 3463-3470.

5. Cusini A, Herren D, Bütikofer L, Plüss-Suard C, Kronenberg A, Marschall J. Intra-hospital differences in antibiotic use correlate with antimicrobial resistance rate in Escherichia coli and Klebsiella pneumoniae: a retrospective observational study. Antimicrob Resist Infect Control, 2018; 7: 89.

6. Laxminarayan, R, Sridhar, D, Blaser, M, Wang, M, Woolhouse, M. Achieving global targets for antimicrobial resistance. Science, 2016; 353: 874-875.

7. Almagor J, Temkin E, Benenson I, Fallach N, Carmeli Y, DRIVE-AB consortium. The impact of antibiotic use on transmission of resistant bacteria in hospitals: Insights from an agent-based model. PLoS One, 2018; 13(5): eo197111.

8. Bengtsson-Palme J, Kristiansson E, Joakim Larsson D. Environmental factors influencing the development and spread of antibiotic resistance. FEMS Microbiol Rev, 2018; 42(1): fuxo53.

9. The Centers for Disease Control and Prevention. Available from: https://www.cdc.gov/antibiotic-use/healthcare/implementation/core-elements.html.

10. Klein E, Van Boeckel T, Martinez E, Pant S, Gandra S, Levin S, et al. Laxminarayana R. Global increase and geographic convergence in antibiotic consumption between 2000 and 2015 . Proc Natl Acad Sci U S A, 2018; 115(15): 3463-3470.

11. Imanpour S, Nwaiwu O, McMaughan D, De Salvo B, Bashir A. Factors associated with antibiotic prescriptions for the viral origin diseases in office-based practices, 2006-2012. JRSM Open, 2017; 8: 1-8.

12. Havers FP, Hicks LA, Chung JR, Gaglani M, Murthy K, Zimmerman R.K, et al. Outpatient Antibiotic Prescribing for Acute Respiratory Infections During Influenza Seasons. JAMA Netw. Open, 2018;1(2): e180243.

13. Zarb P, Amadeo B, Muller A, Drapier N, Vankerckhoven V, Davey P, et al. Identification of targets for quality improvement in antimicrobial prescribing: the web-based ESAC Point Prevalence Survey 2009. J Antimicrob Chemother, 2011; 66: 443-449.

14. Ansari F, Erntell M, Goossens H, Davey P. The European Surveillance of Antimicrobial Consumption (ESAC) point-prevalence survey of antibacterial use in 20 European hospitals in 2006. Clin Infect Dis, 2009; 49: 1496-504.

15. Schroeck J.L, Ruh C.A, Sellick J.Aa Jr, Ott M.C, Mattappallil A, Mergenhagen K.A. Factors Associated with Antibiotic Misuse in Outpatient Treatment for Upper Respiratory Tract Infections. Antimicrob Agents Chemother, 2015; 59: 3848-52. 
16. McDonagh M, Peterson K, Winthrop K, Cantor A, Holzhammer B, Buckley D.I. Improving Antibiotic Prescribing for Uncomplicated Acute Respiratory Tract Infections. Rockville (MD): Agency for Healthcare Research and Quality (US); 2016. Report No.: 15(16)-EHCo33-EF.

17. Zarb P, Coignard B, Griskeviciene J, Muller A, Vankerckhoven V, Weist K, et al. The European Centre for Disease Prevention and Control (ECDC) pilot point prevalence survey of healthcare-associated infections and antimicrobial use. Euro surveill, 2012; 17(46): 20316.

18. Pristaš I, Baršić B, Butić I, Zarb P, Goossens H, Andrašević A.T. PPS on antibiotic use in a Croatian Infectious Disease Hospital. J Chemother, 2013; 25(4): 222-8.

19. Li B, Webster T.J. Bacteria antibiotic resistance: New challenges and opportunities for implant-associated orthopedic infections. J Orthop Res, 2018; 36(1): 22-32.

20. Ayukekbong JA, Ntemgwa M, Atabe AN. The threat of antimicrobial resistance in developing countries: causes and control strategies. Antimicrob Resist Infect Control, 2017; 6: 47.

21. Bhatia R, Katoch VM, Inoue H. Creating political commitment for antimicrobial resistance in developing countries. Indian J Med Res, 2019; 149(2): 83-86.

22. Klein E, Gandra S, Pant S, Mojica N, Martinez E, Laxminarayan R. Global Antibiotic Use and Resistance. Open Forum Infect. Dis, 2016; 3/1: 1487.

23. Global point prevalence survey of antimicrobial consumption. Available from: http://www. global-pps.com.

24. WHO Collaborating Centre for Drug Statistics Methodology, Guidelines for ATC classification and DDD assignment, 2017. Available from: https://www.whocc.no/atc_ddd_ index/?code $=$ Jo1 .

25. Versporten A, Zarb P, Caniaux I, Gros M.F, Drapier N, Miller M, et al. Global-PPS network. Lancet Glob. Health, 2018; 6(6): 619 - 629.

26. Oduyebo O.O, Olayinka A.T, Iregbu K.C, Versporten A, Goossens H, Nwajiobi-Princewill P.I, et al. A point prevalence survey of antimicrobial prescribing in four Nigerian Tertiary Hospitals. Ann Trop Pathol, 2017; 8: 42-6.

27. Mulwahali Wambale J, Liesse Iyamba JM, Masika Mathe D, Kabuyaya Kavuo S, TakaisiKikuni. Point prevalence study of antibiotic use in hospitals in Butembo. Int. J. Med. Med. Sci, 2016; 8: 133-139.

28. Faria S, Sodano L, Dauri M, Sabato AF, Gjata A, Kito I, et al. First point prevalence survey of nosocomial infections in the intensive care units of a tertiary care hospital in Albania. J Hosp Infect, 2007; 65: 244-250.

29. Røder B.L, Nielsen S.L, Magnussen P, Engquist A, Frimodt-Møller N. Antibiotic usage in an intensive care unit in a Danish university hospital. J Antimicrob Chemother, 1993; 32: 633-42.

30. Duo-shuang X, Li-li X, Rui L, Qiao H, Qing-qin L, Wei X. A multicenter point-prevalence survey of antibiotic use in 13 Chinese hospitals. J Infect Public Health, 2015; 8: 55-61.

31. Aghdassi S.J.S, Gastmeier P, Piening B.C, Behnke M, Peña Diaz L.A, Gropmann A, et al. Antimicrobial usage in German acute care hospitals: results of the third national point prevalence survey and comparison with previous national point prevalence surveys. J Antimicrob Chemother, 2018; 73(4): 1077-1083.

32. Morioka H, Nagao M, Yoshihara S, Ohge H, Kasahara K, Shigemoto N, et al. The first multi-centre point-prevalence survey in four Japanese university hospitals. J Hosp Infect, 2018; 99(3): 325-331. 
33. Polk R.E, Fox C, Mahoney A, Letcavage J, MacDougall C. Measurement of adult antibacterial drug use in 130 US hospitals: comparison of defined daily dose and days of therapy. Clin Infect Dis, 2007; 44(5): 664-70.

34. Versporten A, Drapier N, Zarb P, Caniaux I, Gros M.F, Miller M, et al. The Global Point Prevalence Survey of Antimicrobial Consumption and Resistance (Global-PPS): A Worldwide Antimicrobial Web-Based Point Prevalence Survey. Open Forum Infect. Dis, 2015; 2(1): 147.

35. Ider B.E, Clements A, Adams J, Whitby M, Muugolog T. Prevalence of hospital-acquired infections and antibiotic use in two tertiary Mongolian hospitals. J Hosp Infect, 2010; 75(3): 214-9.

36. Skoog G, Struwe J, Cars O, Hanberger H, Odenholt I, Prag M, et al. Skärlund K.; Ulleryd P. Repeated nationwide point-prevalence surveys of antimicrobial use in Swedish hospitals: data for actions 2003-2010. Euro Surveill, 2016; 2: 30264.

37. Liang S.Y, Kumar A. Empiric antimicrobial therapy in severe sepsis and septic shock: optimizing pathogen clearance. Curr Infect Dis Rep, 2015; 17(7): 493.

38. Viasus D, Simonetti A, Garcia-Vidal C, Niubó J, Dorca J, Carratalà J. Impact of antibiotic de-escalation on clinical outcomes in community-acquired pneumococcal pneumonia. J Antimicrob Chemother, 2017; 72(2): 547-553. 